\title{
Linx
}

Revue des linguistes de l'université Paris X Nanterre

$75 \mid 2017$

Imaginaires de la ponctuation. Ordre et inquiétude du discours

\section{Emplois de la virgule en portugais: une réflexion à partir de textes de scripteurs brésiliens}

\section{Geovana Soncin et Luciani Tenani}

\section{CpenEdition}

\section{Journals}

Édition électronique

URL : http://journals.openedition.org/linx/1938

DOI : $10.4000 / \operatorname{linx} .1938$

ISSN : 2118-9692

Éditeur

Presses universitaires de Paris Nanterre

Édition imprimée

Date de publication : 22 décembre 2017

Pagination : 179-199

ISBN : 978-2-84016-308-4

ISSN : 0246-8743

Référence électronique

Geovana Soncin et Luciani Tenani, «Emplois de la virgule en portugais: une réflexion à partir de textes de scripteurs brésiliens », Linx [En ligne], 75 | 2017, mis en ligne le 23 novembre 2018, consulté le 30 avril 2019. URL : http://journals.openedition.org/linx/1938; DOI : 10.4000/linx.1938

Ce document a été généré automatiquement le 30 avril 2019

Département de Sciences du langage, Université Paris Ouest 


\title{
Emplois de la virgule en portugais: une réflexion à partir de textes de scripteurs brésiliens
}

\author{
Geovana Soncin et Luciani Tenani
}

1 Au Brésil, dans l'actuelle configuration de la recherche théorique en linguistique, établir les signes de ponctuation en tant qu'objet d'étude implique d'aborder le rapport entre oralité et écriture. Cette relation, qui traverse différentes études sur la ponctuation, est polémique: d'un côté, il y a des travaux qui soutiennent le rapport entre prosodie et ponctuation et qui thématisent ses analyses à partir d'elle-même (Cagliari, 1989; Pacheco 2003, 2006) ; de l'autre, il y a la tradition grammaticale (Rocha Lima, 1986 ; Luft, 1998 ; Bechara, 1999 ; Cunha \& Cintra, 2001) et les études théoriques délibérément autonomistes (Dahlet, 2002, 2006) ${ }^{1}$ qui, s'appuyant sur Anis (1983), réfutent la relation entre prosodie et ponctuation.

2 Cette polémique s'accentue particulièrement au sujet de la virgule. La complexité de la virgule est avérée, soit par l'instabilité des normes concernant son emploi, soit par les différentes unités qu'elle peut délimiter, soit par les différentes fonctions qu'elle peut exercer, en plus des nombreuses approches qui peuvent la décrire. Dans le courant qui refuse le rapport entre oralité et écriture, pour faire prévaloir la logique grammaticale établissant la systématisation des normes, la virgule est le signe qui témoigne d'incohérences à l'intérieur même du camp grammatical normatif du portugais du Brésil et - peut-être par conséquent - d'une incompréhension chez les élèves. Ces incohérences se révèlent lorsque les grammairiens - même lorsqu'ils présentent les règles d'emploi de la virgule fondées sur des critères syntaxiques - rapportent, dans la présentation des fonctions générales des signes de ponctuation, le besoin inhérent à l'écriture de reconstituer le mouvement de l'élocution orale par le biais des signes de ponctuation (Cunha \& Cintra, 2001; Rocha Lima, 1986); ou, sous un angle plus fonctionnaliste, lorsqu'ils associent les signes de ponctuation au succès de la communication à travers la relation qu'ils établissent avec des aspects rythmiques et mélodiques (Bechara, 1999). Au- 
delà de cette incohérence interne aux affirmations des grammairiens, le désaccord sur la norme exhibe la fragilité de la convention ${ }^{2}$.

Si d'un côté les grammaires du portugais, en homogénéisant et en systématisant l'emploi de la virgule par le biais de normes fondées sur des critères syntaxiques, contribuent fortement à l'établissement d'un système détaché de l'oralité, les incohérences internes et externes de ce système contribuent par ailleurs à maintenir le débat. À propos du français, Rault (2014), dans son étude sur la morphogenèse de la ponctuation dans laquelle il met en question une tendance possible à la grammaticalisation des signes de ponctuation, rapporte que, dans le développement du système de ponctuation, la contradiction entre les critères fondés sur la " pause » et les critères d'ordre logique et grammatical peut être résolue en considérant, à partir des emplois du terme dans un certain nombre d'énoncés définitionnels, que le spectre sémantique de la "pause » inclut en réalité des considérations syntaxiques et sémantiques. En réfutant Barko (1977), Rault (2014 : 3) montre ainsi que le la notion de "pause » ne peut être réduite à la dimension pneumatique. Même s'ils n'avaient pas vraiment cessé de croire aux caractéristiques respiratoires de la ponctuation, de nombreux grammairiens avaient déjà la conscience de la nature logique du système de ponctuation qu'ils élaboraient.

Le constat de Rault (2014) met en évidence une question qui nous paraît fondamentale : la nature hétérogène de la ponctuation, définie par son processus historique de développement, dans lequel les typographes et les grammairiens ont un rôle primordial. Cependant, l'hétérogénéité de la ponctuation est aussi définie par la valeur qu'elle acquiert en tant qu'activité symbolique. C'est ce second aspect que nous nous proposons d'aborder ici, en examinant l'emploi de la virgule dans des textes d'élèves brésiliens, soit des scripteurs dont l'écriture est en voie de conventionnalisation. À travers l'analyse des emplois conventionnels et non conventionnels de la virgule, qu'est-ce que nous révèlent les virgules employées à propos des moyens par lesquels les scripteurs construisent le sens dans l'écriture ? En poursuivant cette question, nous envisageons le sens, les normes et le sujet, dans un ensemble d'imaginaires : les imaginaires de la ponctuation élaborés par ceux qui écrivent.

\section{Fonctions de la virgule et normes d'emploi dans le portugais du Brésil}

5 Malgré les divergences entre grammairiens sur l'emploi de la virgule, les instructions suivantes sont considérées en tant que règles dans le portugais du Brésil: 1) on emploie la virgule pour séparer des éléments de même fonction syntaxique lorsqu'ils ne sont pas liés par des conjonctions additives ; 2) on emploie la virgule pour séparer des phrases juxtaposées ; 3) on doit employer la virgule pour séparer des propositions coordonnées syndétiques à l'exception de celles qui sont liées par la conjonction ; 4) on doit employer la virgule pour séparer des phrases subordonnées adjectives explicatives ; 5) on emploie la virgule pour isoler l'apposition, le vocatif, des éléments et des phrases adverbiales anticipés ou déplacés.

6 En guise de prolongement à ce panorama des normes, quelques instructions précisent où la virgule ne doit pas être employée : entre le sujet et le verbe; entre le verbe et ses compléments ; entre des éléments syntaxiquement liés. 
7 En dehors des grammaires, ces normes sont interprétées en lien avec l'organisation graphique de l'écriture et organisées en deux groupes définis par un fonctionnement commun. Catach (1994) attribue à la virgule deux fonctionnements : « la virgule plus » et « la virgule moins ». Tandis que la première construit des relations de coordination, en séparant des éléments mis côte à côte, la seconde, toujours doublée ou combinée avec un autre signe, marque des hiérarchisations faites à l'intérieur de la phrase, comme dans des cas d'insertion et de modification de l'ordre des éléments.

8 Cette organisation est reprise par Dahlet $(2003 ; 2006)$ qui classe les emplois de virgule selon deux schémas : « virgule en schéma simple » et « virgule en schéma double ». Dans la description du système d'emploi de virgule conçu par Dahlet (2006) pour le portugais, la virgule est classée comme signe de séquence dont la fonction est de délimiter et séparer des unités coordonnées ou ajouter des unités hiérarchiques dans la structure de la phrase : les structures antéposées et les structures détachées. Tandis que la fonction de délimitation et séparation caractérise l'emploi de la virgule en schéma simple, la fonction d'agrégation caractérise la virgule en schéma double. Vues sous le prisme de ces divisions, les instructions normatives se réduisent : celles qui guident l'emploi de la virgule pour séparer des éléments de même fonction syntaxique, des propositions juxtaposées et des propositions coordonnées introduites par une conjonction (sauf et) se justifient par le fait de promouvoir l'identification des limites dans la structuration des unités. L'identification de ces limites est faite par l'emploi de la virgule en schéma simple. À leur tour, les normes qui régissent l'emploi de la virgule pour isoler des propositions subordonnées adjectives explicatives, des appositions, des vocatifs, des éléments et des propositions adverbiaux anticipés ou déplacés, ainsi que d'autres insertions faisant irruption dans la structure et dans l'ordre canonique de la phrase se justifient par le fait de promouvoir la structuration d'unités qui ne se trouvent pas au même niveau syntaxique de la proposition à laquelle ils appartiennent; il s'agit donc d'établir une limite dans la linéarité graphique de la proposition écrite pour identifier différents plans de structuration syntaxique.

Dans son travail Étude de la distribution de la virgule dans les phrases de textes argumentatifs d'expression française, Simard (1993), en abordant le fonctionnement syntaxique de la virgule dans des contextes de coordination et de hiérarchisation, conclut que les virgules indiquent dans la structure syntaxique superficielle des modifications qui ont lieu dans un plan syntaxique plus profond: la structure sous-jacente. Ainsi, en agissant dans la relation entre structure superficielle et structure profonde, les virgules permettent au lecteur de rétablir mentalement la chaîne syntaxique.

Soit dans les grammaires, soit dans les travaux de description fonctionnelle des signes de ponctuation, la virgule est généralement définie en fonction de son comportement syntaxique primordial. Ce comportement est, cependant, et en même temps, une qualité et un facteur de complication. Qualité, car, selon les mots de Védénina, « la virgule est le signe le plus polyvalent de ceux que présente la ponctuation. Elle obéit à des règles imposées par la syntaxe » (1989 : 35-36). Facteur de complication, car, devant suivre la logique de la syntaxe, la virgule est, dans le portugais du Brésil, le signe qui présente, pour les scripteurs, les plus grandes difficultés d'emploi (Soncin, 2014). Une grande partie de cette difficulté provient de la complexité qu'on attribue à la virgule au sein de l'ensemble des signes de ponctuation. Telle est la position de Dahlet (2006), pour qui la virgule est le signe le plus complexe car : 
i. c'est le seul signe qui fonctionne aussi bien dans un schéma double $(/,, /)$ que dans un schéma simple $(/, /)$;

ii. c'est le seul signe qui en dehors de la dimension énonciative, est capable d'opérer simultanément sur deux dimensions, inter-clause et intra-clause ;

iii. il en découle que la /,/ est le signe syntaxique par excellence : [...] le plus apte à fournir, à porter et distribuer les catégories fonctionnelles, aussi bien dans la succession que dans la hiérarchisation (double virgule) ${ }^{3}$.

11 Sans doute, la complexité syntaxique qui accompagne la virgule explique la difficulté d'emploi par les élèves, mais cette complexité est-elle centrale pour comprendre, non le système de ponctuation, mais les moyens par lesquels la virgule est utilisée dans les textes par ces élèves? Et ces moyens peuvent-ils être traduits en termes d'imaginaire?

\section{La question de la pause}

12 Même si la logique syntaxique régit les normes, il n'est pas rare de rencontrer la notion de pause pour évoquer les emplois de la virgule, ce qui, en adaptant les hypothèses de Rault (2014) aux grammaires du Portugais Brésilien, ne serait pas forcément contradictoire. Afin d'apporter des éléments sur le statut de la pause et son importance pour l'analyse de la virgule, reprenons quatre importants grammairiens du portugais moderne.

Dans leur Nova Gramática do Português Contemporâneo, Cunha \& Cintra (2001) considèrent la virgule, le point et le point-virgule comme des "signes qui marquent surtout la pause ", mais précisent que ces signes peuvent aussi désigner des modifications mélodiques. Ainsi, « la virgule indique que la voix reste en suspens, en attendant que la phrase se complète » 4 .

Rocha Lima (1986) ouvre le chapitre destiné aux signes de ponctuation de sa Gramática Normativa da Língua Portuguesa, en présentant une division de ce qu'il appelle "pauses rythmiques ": des pauses qui ne cassent pas la continuité du discours, des pauses qui indiquent la fin du discours ou d'une de ses parties et des pauses qui servent à mettre en évidence une intention ou un état émotif. L'organisation des signes de ponctuation est donc établie par le grammairien en se basant sur cette classification des pauses, dans laquelle la virgule marquerait le premier type de pause à l'écrit, à côté du tiret, des parenthèses, du point-virgule et des deux points.

15 Bechara (1999), en s'autorisant de manière explicite de Catach (1994) pour développer le chapitre sur la ponctuation de la Moderna Gramática Portuguesa, la conçoit comme un système de renforcement de l'écriture guidé par la syntaxe, qui a pour fonction d'organiser des parties du discours et de mettre en relief des fonctions syntaxiques, intonationnelles et sémantiques. Dans cette perspective, la virgule est considérée un signe de pause non conclusive, dont la fonction est de signaler la suite de la phrase dans laquelle elle se trouve.

16 Luft (1998), enfin, dans A vírgula, livre d'instructions normatives pour l'emploi de la virgule, affirme catégoriquement que la ponctuation suit des critères syntaxiques et non prosodiques et pense que la plupart des fautes de ponctuation sont motivées par le lien entre virgule et pause. Néanmoins l'auteur se contredit en affirmant que la virgule représente beaucoup plus les tonalités de la parole que la pause.

17 La mention de la pause chez tous les grammairiens montre son importance quant à l'emploi de la virgule. Cette importance invite à s'interroger sur les relations entre pause 
et virgule, mais aussi sur le statut même de la notion de pause. Sa permanence dans un discours qui fait de la syntaxe le fondement des règles est, à nos yeux, un indice de sa valeur pour comprendre la nature de la virgule, même si elle ne n'est pas associée au système qui la régit. Pour cela, nous considérons précieux le questionnement lancé par Julien Rault :

Il peut paraître alors pertinent de s'interroger sur le véritable spectre sémantique du terme " pause ». Si les grammairiens maintiennent le critère pausal dans leur définition mais élaborent dans le même temps un système fondé sur la syntaxe et la logique, c'est peut-être que le terme " pause » est, en définitive, beaucoup plus polyvalent qu'on a bien voulu le croire. (2014:3)

Avec ce questionnement, Rault avance l'hypothèse que le terme " pause » a un spectre sémantique plus large que celui qui s'appuie sur l'idée d'intervalle pour la respiration. Parmi les possibilités sémantiques pour le terme " pause ", l'auteur retrouve les notions de « suspension du sens " ${ }^{5}$, tout en observant que, par opposition au terme " intonation », la pause est fréquemment employée pour évoquer la distinction des parties du discours, devenant, ainsi, pas uniquement « une notion orale » $(2014: 4)$. De cette manière, Rault considère que, si la notion de pause a acquis d'autres traits différents de la pure réalisation phonétique pour la définition des signes de ponctuation, il lui a été attribué, dans ce processus de systématisation logique de la ponctuation, une valeur grammaticale. L'évaluation de la pause comme notion qui acquérant une valeur grammaticale et participant d'une réflexion linguistique sur la virgule, sur un imaginaire ponctuant, est un facteur fondamental pour l'analyse que nous proposons. Cependant, nous apportons à la discussion la notion de « limite », qui pourrait inclure la valeur grammaticale attribuée à la pause.

Soit dans l'approche qui la conçoit comme intervalle pour la respiration, soit dans celle qui la considère comme une suspension du sens ou une distinction de parties du discours et même jusqu'à ceux qui l'entendent comme un moment de silence, la pause est toujours une limite. Limite entre respirer et ne pas respirer, entre ce qui se dit et ce qui est à dire, entre les différentes parties du discours. En tant que limite, donc, elle acquiert une valeur d'organisation et structuration du dire, car, d'une part, elle sépare ce qui est différent, d'autre part, elle produit l'effet d'unir les différences dans l'ensemble final.

Dans les grammaires du portugais auxquelles nous avons fait référence, l'idée de la virgule comme signe pausal responsable de l'organisation structurale de la proposition ou du texte est sensible, chez Cunha \& Cintra (2001), lorsque la virgule est présentée comme signe qui met la voix en suspens pour que la proposition se complète; chez Rocha Lima (1986), cela est perceptible au moment où la virgule est définie en tant que signe marquant une pause qui, même insérée dans le discours, ne casse pas sa continuité ; chez Bechara (1999), cela se perçoit lorsque la virgule est liée au terme confus " pause inconclue » dont la fonction est d'indiquer la suite de la phrase. Chez Luft (1998), à son tour, le critère pausal est nié pour donner la priorité à la structuration; nous voyons, selon le regard des autres grammairiens, que la pause, au contraire, entendue comme limite, ne nie pas l'organisation structurale.

Il est possible de considérer l'organisation structurale de la langue du point de vue prosodique pour vérifier quel serait le statut de la pause dans cette organisation. Dans le modèle phonologique connu comme Phonologie Prosodique (Nespor \& Vogel, 1986), la langue se structure, sur le plan prosodique, en différentes unités qui s'organisent hiérarchiquement et qui gardent les relations entre elles. Chacune de ces unités 
présuppose une représentation phonologique formée à partir de catégories advenues de la syntaxe. Dans ce modèle, l'unité prosodique qui nous intéresse pour penser la question de la pause est ce qu'on appelle "phrase intonationnelle ». En considérant que, quand nous parlons, nous n'utilisons pas des propositions isolées, notre parole est, alors, structurée prosodiquement en phrases intonationnelles placées côte à côte. Dès lors, dans l'association entre phrases intonationnelles, la limite ne marque pas seulement le changement de contour, c'est-à-dire, la fin de l'une et le début de l'autre, mais aussi la possibilité de marquer une pause. Sous la perspective prosodique, la pause a lieu dans la position limite, dans la position où, dans le domaine de la phrase intonationnelle, se trouve ce qu'on appelle une "frontière prosodique ", concept primordial du modèle pour l'identification de ses unités. Si nous envisageons la prosodie à partir de ce prisme structural, plus qu'un ensemble de réalisations phonétiques possibles qui varie en fonction des conditions d'énonciation, la notion de pause ne reste pas cloitrée à l'idée de respiration dans la réalisation de la parole, mais au concept de frontière qui, avant tout, structure la langue. L'analyse qui suit met en relation la structuration de la langue promue par l'établissement de frontières prosodiques et la structuration du texte écrit promue par l'emploi des virgules. À travers cette analyse, nous démontrons comment cette relation formelle ne se détache pas des significations produites dans le discours et comment elle est révélatrice des imaginaires conditionnant les emplois de la virgule.

\section{Un moyen d'appréhender le fonctionnement discursif des emplois de la virgule}

22 Notre analyse se fonde sur les réflexions entreprises par Soncin (2014). Visant à investiguer les possibles relations entre frontière prosodique et emploi de virgules, l'auteur a vérifié que $93,4 \%$ des virgules non-conventionnelles et $100 \%$ des virgules conventionnelles employées dans 284 textes l'ont été dans des positions où l'on reconnaît des frontières prosodiques, notamment des frontières de phrase intonationnelle. Face à ce résultat quantitatif et par le biais d'une méthodologie qui a tenu compte des significations des virgules employées à l'intérieur des textes analysés, Soncin soutient que les virgules marquent dans le texte écrit la fonction délimitatrice des frontières prosodiques, essentielle à la fois pour la structuration et la signification du dire. Ainsi, la frontière prosodique fonctionne comme l'indice de l'existence d'une unité prosodique sous-jacente à la structuration de la langue - tout comme il existe les unités syntaxiques et, en tant que telle, a une valeur aussi bien pour l'organisation formelle des énoncés que pour l'organisation des sens produits en eux.

23 Nous défendons la thèse de l'organisation formelle et de l'organisation des sens que la virgule exerce sur l'écriture en délimitant des frontières; alors, nous cherchons à identifier la manifestation de ces processus d'organisation du texte à travers l'emploi de la virgule. Les textes des élèves se caractérisent par des oscillations entre emplois conventionnels et emplois non conventionnels de virgule. Dans l'analyse de ces emplois, nous avons privilégié deux axes: (i) d'un côté, l'organisation formelle du texte, en établissant la relation entre les frontières délimitées (syntaxique et prosodique) et l'imaginaire du bon usage de l'emploi de la virgule établi par la prescription normative; (ii) d'un autre côté, l'organisation des sens, interprétant les frontières prosodiques marquées par la virgule comme des points du texte dans lesquels on observe des divisions de filiations discursives et de positions-sujet dans le processus de textualisation du 
discours (Orlandi, 2007). Nous comprenons, avec Orlandi (2007), que le discours, toujours incomplet, est l'effet de sens entre locuteur et interlocuteur, tandis que le texte est l'unité empirique du discours qui, délimitée par une extension, a imaginairement un début, une fin et un auteur qui définit son origine. De ce fait, " il y a un long parcours entre l'interdiscours (mémoire du dire) et le texte. Il faut poser un processus de textualisation organisant le rapport obligé du réel discursif à l'imaginaire textuel » (Orlandi, 2007 : 66). Ce parcours est nommé de " mise-en-texte du discours » ou « textualisation du discours ». Considérant qu'il y a toujours une lacune entre l'unité textuelle (imaginaire) et la discursivité, suivons Orlandi qui conceptualise la ponctuation comme mécanisme pour créer l'illusion de l'unité textuelle, face à la dispersion discursive :

Assurant l'effet pragmatique d'un monde sémantiquement normal et d'un texte qui a son unité dans la dispersion, la ponctuation sert à conférer au discours une dimension textuelle. Mécanisme de spatialisation du sens sur la surface du texte - le sens étant du point de vue discursif foncièrement inépuisable - la ponctuation est une violence symbolique nécessaire: un mécanisme qui administre notre rapport à incomplétude du langage, travaillant l'incomplétude du sujet et l'inachèvement $d u$ sens. C'est l'espace symbolique des rapports de sens qui est ponctué: le point fonctionne imaginairement comme signe d'un achèvement (impossible). La ponctuation administre - sans les éliminer - le manque et l'équivoque. (2007: 70) signe de ponctuation: la frontière délimitée par la virgule serait donc un espace symbolique de rapports de sens; mais elle est également un espace symbolique de relations formelles concernant la formulation linguistique du discours, car nous croyons que les sens produits dans le discours se constituent au cœur des pratiques d'emploi du langage (Bakhtine, 1984 ; Corrêa, 2004) et, par conséquent, sont aussi définis en fonction des différentes façons de production du dire. Corrêa (2001) défend l'hétérogénéité du processus de production des énoncés, en particulier des énoncés écrits. Selon lui, dans le processus d'écriture d'un texte, le sujet construit des images de son propre acte d'écrire et produit des sens à l'écrit. La construction d'images a lieu, car le sujet qui écrit est, avant tout, un sujet du langage qui transite (et se constitue) par des pratiques sociales qui projettent des signifiés autant par le biais de l'oralité que par celui de l'écriture. L'ambition de Corrêa (2004) est donc de considérer l'écrit comme un processus de constitution hétérogène ${ }^{6}$. L'hétérogénéité de l'écrit est montrée par le transit des écrivains par différents moyens de représenter l'écrit : dans certaines marques du texte, il est possible de constater une image de l'écrit fidèle à l'oralité, tandis que d'autres marques (dans les mêmes textes, des mêmes écrivains) dénoncent l'image que les écrivains font de l'écrit en tant que code écrit institutionnalisé et autonome par rapport à l'oralité. Ce transit se justifie par ce que Corrêa (2004) appelle dialogisme avec le déjà parlé / écrit, en référence aux discours, dans ses modes parlé et écrit de manifestation de la langue, qui constituent le sujet.

Dans l'analyse des emplois de virgule, en évaluant l'organisation formelle et des sens des énoncés, établie par les frontières délimitées par la virgule, nous faisons émerger des éléments qui nous permettent de regarder vers les imaginaires créés sur l'écrit. Ainsi, nous nous approprions des imaginaires sur l'écrit, proposés par Corrêa (2004), pour penser les imaginaires sur l'emploi de virgule construits dans le processus de textualisation du discours, qui sont

un recours pour capter le mode hétérogène par lequel se donne la production des sens dans les énoncés, c'est-à-dire, pour capter des moments où les sujets s'appuient sur des discours produits par des pratiques lettrées (je les limite, dans ce 
point, à celles énoncées par le biais de l'écrit), ou dans des discours produits par des pratiques orales (je les limite, dans ce point, à celles énoncées par le biais de la parole) $)^{7}$. entre 13 et 14 ans, appartenant à la dernière année de l'Enseignement Fondamental au Brésil. Ces textes appartiennent à la Base de Données de Productions Écrites de l'Enseignement Fondamental, organisée à travers des financements de la Fondation de Soutien à la Recherche de l'État de São Paulo - FAPESP (Processus: 2013/14546-5 et 2009/14848-6) - et disponible gratuitement à l'UNESP'.

\section{Virgules et marques du rapport du sujet avec la textualisation du discours : imaginaires sur la ponctuation}

Les emplois de la virgule peuvent être considérés aussi bien comme des emplois conventionnels que non conventionnels. Caractérisés par la présence de la virgule, les emplois que nous avons analysés font référence aux cas où la virgule a été employée dans le texte et non aux cas où la virgule n'a pas été employée, même si elle était requise par convention.

Le choix de privilégier les emplois non conventionnels se justifie par notre désir d'entrevoir les imaginaires construits pendant le processus de textualisation. Les emplois non conventionnels n'étant pas « camouflés » dans le standard du bon usage peuvent être révélateurs du rapport du sujet à l'écrit (son écrit) et, par conséquent, laissent en évidence l'incomplétude de cette relation. L'organisation (de forme et de signifié) que le scripteur a imprimé sur son texte par le biais des virgules révèle quelque chose des imaginaires de l'emploi de la virgule à l'écrit.

(1) E se um dia nós os brasileiros precisarmos da Amazônia, nós vamos ficar na mão, porque aí os estrangeiros já vão ter tomado tudo o que é nosso. Alguém competente , tem que por fim nisso?.

En (1), les deux premières virgules sont conventionnelles, car elles marquent deux frontières syntaxiques, caractérisées par les normes, respectivement, comme une proposition adverbiale anticipée et le début d'une proposition coordonnée explicative. En même temps, ces deux virgules balisent des frontières prosodiques, une fois que chacune des trois propositions délimitées par les virgules se configurent, structuralement, en tant que phrases intonationnelles. La troisième virgule est d'emploi non conventionnel, car la frontière entre sujet et verbe ne constitue pas une frontière syntaxique qui, du point de vue de la norme, doive être marquée par virgule. Cependant, sous le prisme de la structuration prosodique, dans la position où elle se trouve, il peut y avoir une frontière de phrase intonationnelle si on considère que le sujet de la proposition peut être focalisé.

Il s'agit en effet de l'extrait d'un texte dans lequel le scripteur aborde la question de l'internationalisation de l'Amazonie, la principale forêt du Brésil. L'auteur évalue la possibilité pour le Brésil de ne plus compter sur l'Amazonie et conclut que l'internationalisation de la forêt doit être bloquée. Cependant, le scripteur, par méfiance à l'égard des autorités brésiliennes, met en relief la nécessité d'une personne pour prendre cette décision, par opposition à l'idée d'inaptitude et inefficacité généralement associée à ceux qui font ce travail au Brésil. Ce sens apporté à l'énoncé, même s'il n'est pas dit 
explicitement, est interprétable si nous tenons compte de l'existence d'une frontière prosodique après le sujet, dans la position où a lieu l'emploi non conventionnel de la virgule : la virgule indique une frontière prosodique et aurait ainsi la fonction de baliser la focalisation du sujet, projetant, ainsi, un sens et pas l'autre. Différemment des deux virgules précédentes où le sujet, pris par l'imaginaire de l'écrit institutionnalisé, répond au standard établi par les normes, la troisième virgule montre que l'écrit a été imaginé par le scripteur comme une représentation fidèle de certaines pratiques de l'oralité dans lesquelles l'accent prosodique construit des sens parfois interdits en forme de mots.

31 Ainsi, les deux premières virgules établissent des frontières syntaxique-prosodiques qui répondent au standard du bon usage de la langue et, en même temps, balisent des conditions de réalité possibles selon la position de l'écrivain (l'internationalisation de l'Amazonie est une menace). La virgule non conventionnelle délimite une frontière prosodique qui réorganise les sens de la proposition, en dirigeant la position mise en place par le scripteur face à la dispersion du discours. Cette virgule est invalidée par la convention: la déviance à l'égard du bon usage de la ponctuation indique l'ancrage du sujet dans des pratiques d'oralité significative pour la production de sens à l'écrit. Considérons l'exemple (2):

(2) Ao contrário de um brasileiro comum ou normal, eu sou a favor, da internacionalização da Amazônia. [...] Por que não deixar que internacionalize a Amazônia? Para mim a resposta seria: "Perda de tempo, grande parte da Amazônia já é usada para as necessidades de outros países" ${ }^{10}$.

Sur un thème identique, l'énoncé a été extrait d'un texte où l'auteur est favorable à l'internationalisation. L'image que le scripteur possède de son propre positionnement est vue comme atypique, car, selon lui, sa prise de position est contraire à celle d'un brésilien commun. La première virgule, conventionnelle, délimite une proposition adverbiale antéposée et une frontière de phrase intonationnelle, respectivement, selon les points de vue syntaxique et prosodique. Simultanément, la virgule balise, sur le plan de l'organisation des sens, la division entre le positionnement de la majorité des Brésiliens qui s'aligne sur les discours de protection du patrimoine national et la position différente et critique prise par le scripteur. On souligne, donc, par le biais de cette virgule conventionnelle, l'abîme discursif entre les deux positions. La prise de position favorable à l'internationalisation est suivie d'une virgule non conventionnelle (deuxième virgule). Si on considère que l'explicitation de ce positionnement est fondamentale, puisqu'elle présuppose comme destinataire préférentiel quelqu'un d'opinion contraire, la virgule acquiert un caractère de marque de relief : marque qui met en avant la position favorable à l'internationalisation, qu'on assume face à la dispersion du discours. En dehors d'une frontière syntaxique et, donc, en dehors du standard établi par la convention, la virgule se trouve, par opposition, à la frontière d'une phrase intonationnelle, dans laquelle la possibilité de récupération d'une emphase prosodique au moment de la lecture du texte produit un effet argumentatif d'imposition et d'éclaircissement. En s'ancrant donc dans des pratiques d'oralité où le recours à l'angle prosodique fonctionne en tant que positionnements (illusoirement) incontestables - comme il arrive, par exemple, dans des débats politiques - le sujet emploie la virgule comme marque graphique qui indique que la projection argumentative de sa prise de position a été imaginée au moment de l'écriture. conventionnel puisqu'il est à la frontière syntaxique de deux unités juxtaposées. Du point 
de vue prosodique, la virgule se situe aussi à une frontière prosodique, une fois que chaque unité de la juxtaposition se configure en deux phrases intonationnelles qui se placent côte à côte. Si nous considérons encore le processus de textualisation du discours, la virgule désigne une frontière entre la conclusion du scripteur - empêcher l'internationalisation de l'Amazonie serait une perte de temps - et la justification de cette conclusion : l'Amazonie est déjà utilisée par d'autres pays. Le respect des normes dans l'emploi de cette dernière virgule, ainsi que dans l'emploi de la première, souligne la standardisation de l'écriture du sujet, mettant en évidence son ancrage dans des pratiques d'usage de l'écriture institutionnalisée. Cependant, la délimitation simultanée de frontières prosodiques et frontières de sens, effectuée par ces virgules, conjointement avec la délimitation de frontières syntaxiques, met en avant le fait que, dans le processus de textualisation, lorsqu'on emploie la virgule, on fait face à l'incomplétude du sujet, du langage et du sens (Orlandi, 2007). Pour cette raison, on peut observer le caractère hétérogène de la ponctuation, parce que même si, dans la surface du texte, transparaît un idéal d'homogénéité créé par convention avec l'établissement de normes, l'usage des signes de ponctuation mobilise différents dimensions linguistiques, montrant qu'elle n'est pas homogène. Considérons, enfin, l'exemple (3):

(3) Aquecimento global, fim do mundo ou catástrofes da natureza. Cada um nomeia como pode, não importa, o que importa de verdade é o problema que não agrava só uma cidade ou então um país mas sim o mundo inteiro. Eu dou o nome de aquecimento global a esse problema, o que está acontecendo é, o excesso de $\mathrm{CO} 2$ (gás carbônico) que as empresas emitem no ar e consequentemente falta $\mathrm{O} 2$ (oxigênio) para o $\mathrm{ar}^{11}$.

Extrait d'un texte qui aborde le pouvoir destructif de l'homme sur la planète, l'énoncé évoque le processus de nomination de l'épuisement de ressources de la Terre. Avant d'arriver à une dénomination finale, le texte montre la propre dispersion discursive en dressant de noms différents pour le processus - réchauffement global, fin du monde ou catastrophes de la nature - qui caractérisent différentes positions discursives. Les trois premières virgules, conventionnelles, ont été employées à l'intérieur de cette dynamique. En délimitant le premier élément d'une énumération, la première virgule non seulement se place à la frontière syntaxique entre deux éléments de l'énumération, mais se place aussi à la frontière prosodique qui sépare ces éléments en différentes phrases intonationnelles $^{12}$ et à la frontière qui divise deux positions discursives: celle qui dénomme scientifiquement le processus réchauffement global et celle qui le dénomme croyance en la fin du monde. Les deuxième et troisième virgules délimitent des propositions coordonnées juxtaposées dont les sens réitèrent la multiplicité de positions discursives sous laquelle le processus de destruction de la Terre peut être traité. Ainsi, si syntaxiquement et prosodiquement il $\mathrm{y}$ a des frontières où les virgules ont été insérées par le fait de délimiter des propositions coordonnées ${ }^{13}$, sur le plan discursif, la deuxième délimite l'incapacité du sujet à présenter tous les noms possibles - « chacun le nomme comme il peut "-, tandis que la troisième délimite l'illusion de contrôle que le sujet projette dans son texte face à la dispersion de discours, en indiquant que la variété de noms n'a pas d'importance. La quatrième virgule employée après la proposition « je donne le nom de réchauffement global à ce problème " est conventionnelle pour délimiter une proposition coordonnée. De cette façon, de même que les deux virgules précédentes, elle désigne une frontière aussi bien syntaxique que prosodique. Face à la variabilité, le sujet met en place une frontière dans la dispersion discursive et définit le problème traité comme réchauffement global. La dernière virgule de l'extrait, employée 
après la forme verbale est, ne délimite pas une frontière syntaxique reconnue ; il s'agit d'un emploi non conventionnel puisqu'elle met en relation des éléments syntaxiquement reliés comme sujet et verbe ou verbe et compléments. En revanche, dans la position où la virgule a été employée, il y a une frontière prosodique: on doit considérer qu'une structure de suspension a été établie entre l'annonce et l'explication de ce qui est le réchauffement climatique et que chacune de ces parties - l'annonce et l'explication - est structurée comme une phrase intonationnelle. Dans cette frontière prosodique délimitée par la virgule - où les deux points pourraient avoir été employés -, la réalisation de la pause est une possibilité dans la lecture qui accentuerait la suspension de l'explication scientifique donnée au processus dénommé antérieurement comme réchauffement global. En s'ancrant, donc, dans l'imaginaire d'un écrit représentant fidèlement l'oralité, le sujet fait appel à la suspension du sens renforcée par la pause dû à sa mémoire de sujet du langage qui a pris contact - directement ou indirectement - avec des pratiques d'oralité où la suspension fonctionne comme un moyen argumentatif ou expositif afin de garder l'attention des interlocuteurs.

L'oscillation entre virgules conventionnelles et non conventionnelles montre le cheminement du scripteur par des imaginaires concernant le processus de constitution de l'écrit. Tantôt répondant à la prescription normative tantôt s'en éloignant, parfois se ralliant à un discours, parfois se ralliant à un autre, le sujet viabilise des moyens pour que l'analyste du langage puisse voir les chemins qu'il a parcourus dans le processus de textualisation du discours et, par conséquent, constater que la complexité du sujet du langage définit des relations symboliques effectuées à l'écrit en employant les signes de ponctuation, plus spécifiquement la virgule.

\section{Conclusion}

Les emplois de la virgule analysés ont été extraits de textes produits par des élèves en salle de classe. Il s'agit donc de textes d'élèves qui sont dans une étape de conventionalisation de l'écrit. Ceci explique l'oscillation entre des emplois conventionnels et non conventionnels, mais aussi le poids qu'acquiert l'encadrement des normes aussi bien pour les élèves, qui veulent atteindre le standard, que pour les professeurs, qui les évaluent selon le standard.

Il advient dans cette quête d'une bonne écriture - caractérisée, pour les virgules, par la logique syntaxique -, des rapports de forme et de sens inusités qui apparaissent dans quelques emplois non par l'intention pragmatique du sujet, mais par sa constitution historique, qui l'oblige à faire face à l'équivoque et au manque qui constituent également le sujet et le langage. La ponctuation est « un mécanisme qui administre notre rapport à l'incomplétude du langage, travaillant l'incomplétude du sujet et l'inachèvement du sens " (Orlandi, 2007); en s'appuyant sur l'analyse des emplois de virgule, (i) l'incomplétude du langage se vérifie, lorsque, dans le processus de textualisation du discours, le langage, même s'il se manifeste sur le produit final soit comme écrit soit comme oral, produit des signifiés dans le rapport entre ses deux modes de manifestation; (ii) l'incomplétude du sujet se montre, donc, au moment où il écrit, ce sujet n'étant pas seulement le sujet de l'écriture, mais aussi, de manière conflictuelle, le sujet de l'oralité par son histoire dans les pratiques de langage qu'il porte avec lui en énonçant; et (iii) l'incomplétude du sens se montre dans la mesure où il n'est pas constitué uniquement par ce qui a été contemplé 
à l'intérieur de l'unité textuelle (imaginaire), mais aussi et forcément par ce qui est resté dans l'univers extérieur.

Ainsi, en comparant les emplois de la virgule, à partir de cette place théorique qui considère l'incomplétude, et les normes dressées par les grammairiens, qui réglementent le standard, nous observons une lacune entre la réglementation et la nature de l'emploi de la virgule à l'écrit. Comme s'il y avait un écart entre l'idéal d'homogénéité syntaxique, qui caractérise les normes, et la réalité hétérogène de l'activité du langage, spécifiquement dans sa forme écrite, qui a dans les signes de ponctuation un repère visible pas seulement d'un processus historique impur (Jenny, 2012), dans lequel les rapports avec l'oralité s'imposent de manière significative, mais aussi un repère visible de l'incomplétude du rapport entre sujet et écriture qui a lieu dans le processus d'écrire. L'histoire du sujet dans le langage est autant dans l'emploi de l'oralité que dans l'emploi de l'écrit, lesquels constituent les sens produits dans un texte. Soulignons l'importance de la notion de frontière prosodique pour la construction d'un imaginaire de l'emploi de virgules, qui

délimite des unités prosodiques qui composent significativement et formellement l'écrit, avec la distinction que les emplois non conventionnels sont identifiés plus par leur caractère iconique pour composer les signifiés tandis que les emplois conventionnels sont identifiés plus par leur caractère formel d'organisation de l'écriture ${ }^{14}$.

Les emplois non conventionnels se caractérisent par leur caractère iconique ${ }^{15}$, car, en désignant des sens véhiculés par la prosodie dans certaines pratiques d'oralité, paradoxalement, ils montrent l'incomplétude du langage, du sujet et du sens. Les emplois conventionnels de la virgule se caractérisent davantage par leur caractère de représentation symbolique, car, en témoignant des moments où les sujets suivent le standard établi par la convention, ils attribuent à l'action de ponctuer par la virgule un statut d'élaboration formelle de l'énoncé, dans lequel les incomplétudes sont camouflées par l'homogénéité du système de normes.

\section{BIBLIOGRAPHIE}

ANIS, J., 1983, « Pour une graphématique autonome ", Langue française, 59, Le signifiant graphique, Larousse, Paris, p. 31-44.

AUTHIER-REVUZ, J., 1982, « Hétérogénéité montrée et hétérogénéité constitutive, éléments pour une approche de l'autre dans le discours », DRLAV, 26, p. 91-151.

BAKHTINE, M., 1984, Esthétique de la création verbale, Gallimard, Paris.

BARKO, I., 1977, «Contribution à l'étude de la ponctuation française au XVII ${ }^{\mathrm{e}}$ siècle (Problèmes de méthode - La ponctuation de Racine) », dans CATACH, N., TOURNIER, C., PETIT, J., (éds), La Ponctuation, recherches historiques et actuelles, tome 2, Paris et Besançon: CNRS et Groupement de recherches sur les textes modernes.

BECHARA, E., 1999, Moderna Gramática Portuguesa, Lucerna, Rio de Janeiro. 
CAGLIARI, L. C., 1989, « Marcadores prosódicos na escrita », Estudos linguísticos, XVIII Anais de Seminários do GEL, Lorena, p. 195-203.

CATACH, N., 1994, La Ponctuation, Presses Universitaires de France, Paris.

CORRÊA, M. L. G., 2004, 0 modo heterogêneo de constituição da escrita, Martins Fontes, São Paulo.

CUNHA, C. F. da, CINTRA, L., 2001, Nova gramática do português contemporâneo, Nova Fronteira, Rio de Janeiro.

DAHLET, V., 2002, « A pontuação e sua metalinguagem gramatical. », Estudos da Linguagem, 10, Faculdade de Letras da UFMG, Belo Horizonte, p.29-41.

DAHLET, V., 2003, Ponctuation et énonciation, Ibis Rouge Editions, Guadelupe.

DAHLET, V., 2006, As (man)obras da pontuação: usos e significações, Associação Editorial Humanitas, São Paulo.

DEMONET, M.-L., 2011, « Ponctuation spontanée et ponctuation civile. » dans La ponctuation à la Renaissance, Paris, Classiques Garnier, p. 129-147.

DOMERGUE, F.-U., 1778, Grammaire française simplifiée ou Traité d'ortographe avec des notes sur la prononciation et la syntaxe, des observations critiques et un nouvel essai de prosodie, Lyon, chez l'auteur.

JENNY, L., 2012, « Mises au point. » Critique, 785, Minuit, p.821-830.

LUFT, C. P., 1998, A vírgula: considerações sobre o seu ensino e o seu emprego, Ática, São Paulo.

NESPOR, M. \& VOGEL, I., 1986, Prosodic Phonology, Foris Publications, Dordrecht.

ORLANDI, E., 2007, « Un point c'est tout », dans AUTHIER-REVUZ, J., LALA, M.-C. (éds), Figures

d'ajout. Phrase, texte, écriture, Paris, Presses Sorbonne Nouvelle, p. 65-77.

PACHECO, V., 2003, Investigação fonético-acústico-perceptual dos sinais de pontuação enquanto marcadores prosódicos, Mestrado em Linguística, Instituto de Estudos da Linguagem, Universidade Estadual de Campinas, Campinas/São Paulo.

PACHECO, V., 2006, O efeito dos estímulos auditivo e visual na percepção dos marcadores prosódicos lexicais e gráficos usados na escrita do português brasileiro, Doutorado em Linguística, Instituto de Estudos da Linguagem, Universidade Estadual de Campinas, Campinas/São Paulo.

RAULT, J., 2014, « De la "pause" à la "valeur" en langue: grammaticalisation des signes de ponctuation? », Congrès Mondial de Linguistique Française, Université Libre de Berlin, p. 19-24.

ROCHA LIMA, L. H., 1986, Gramática Normativa da Língua Portuguesa, José Olympio, Rio de Janeiro.

SIMARD, M., 1993, Étude de la distribution de la virgule dans les phrases de textes argumentatifs d'expression française, Master, Université Laval, Québec.

SONCIN, G. C. N., 2014, Língua, discurso e prosódia: investigar o uso da vírgula é restrito? Vírgula!, Doutorado em Estudos Linguísticos, Instituto de Biociências, Letras e Ciências Exatas, Universidade Estadual Paulista, São José do Rio Preto/São Paulo.

VÉDENINA, L. G., 1989, Pertinence linguistique de la présentation typographique, Peeters/Selaf, Paris. 


\section{NOTES}

1. Dans ce même encadrement théorique, Dahlet a publié en France, en 2003, l'ouvrage Ponctuation et énonciation, en abordant la langue française, dans lequel il est possible de voir des exemples en français.

2. Voir Soncin (2014) pour l'analyse approfondie des différences dans la présentation des normes d'emploi de la virgule dans quatre grammaires sélectionnées du Portugais.

3. Nous traduisons le texte en portugais : «i. é o único sinal que funciona tanto num esquema duplo $(/,, /)$ quanto num esquema simples $(/, /)$; ii. é o único sinal que independentemente da dimensão enunciativa, é capaz de atuar simultaneamente em duas amplitudes, intercláusulas e intracláusula; iii. decorre disso que a /,/ é o sinal sintático por excelência: o mais construtor de sintaxe, o mais apto para fornecer, carregar e distribuir as categorias funcionais, tanto na sucessão quanto na hierarquização (dupla vírgula). » (Dahlet, 2006 : 143).

4. Nous traduisons le texte portugais suivant : « a vírgula assinala que a voz fica em suspenso, à espera de que o período se complete. » (Cunha \& Cintra, 2001 : 654).

5. Selon la description de Rault (2014), la notion de « repos » présentée par Domergue (1778) fait cohabiter la valeur sémantique « suspension du sens » et la valeur prosodique « repos ", indiquant la polyvalence acquise par la pause pour l'auteur à travers l'association de sens + repos.

6. La théorie de l'hétérogénéité développée par Authier-Revuz (1982) et le concept de dialogisme, de Bakhtine (1984), sont des guides pour le développement du mode hétérogène de constitution de l'écrit de Corrêa (2004).

7. Nous traduisons le texte portugais suivant : «um recurso para captar o modo heterogêneo pelo qual se dá a produção dos sentidos nos enunciados, ou seja, para captar momentos em que os sujeitos se apoiam em discursos produzidos por práticas letradas (restrinjo-as, neste ponto, àquelas enunciadas por meio da escrita), ou em discursos produzidos por práticas orais (restrinjo-as, neste ponto, àquelas enunciadas por meio da fala). » (Soncin, $2014:$ 92).

8. La banque de données dispose de 5.284 textes de différents genres produits par 662 élèves de l'âge entre 10 et 14 ans, appartenant aux dernières quatre années du système d'Enseignement Fondamental au Brésil, disponible sur: http:// www.convenios.grupogbd.com/redacoes/Login. Le matériel qui compose la banque de données a été constitué à partir du Projet d'Extension Universitaire "Ateliers de Lecture, Production et Interprétation de Texte" réalisé par l'UNESP, dans la ville de São José do Rio Preto - São Paulo, en partenariat avec une école publique de la même ville. Le projet a été développé pendant les années 2008 et 2011, sous la coordination des professeurs Luciani Ester Tenani et Sanderléia Roberta Longhin-Thomazi, et il a bénéficié d'un financement du Département d'Extension du Rectorat de l'UNESP.

9. Traduction : «Et si un jour nous les Brésiliens avons besoin de l'Amazonie, nous serons livrés à nous-mêmes, parce que là les étrangers auront déjà pris tout ce qui est à nous. Quelqu'un de compétent doit mettre fin à ça. »

10. Traduction : «Au contraire d'un Brésilien commun ou normal, je suis pour l'internationalisation de l'Amazonie. [...] Pourquoi ne pas accepter qu'on internationalise 
l'Amazonie ? Pour moi la réponse serait: "Perte de temps, une grande partie de l'Amazonie est déjà utilisée pour les besoins d'autres pays" ".

11. Traduction : "Réchauffement global, fin du monde ou catastrophes de la nature. Chacun le nomme comme il peut, peu importe, ce qui importe vraiment c'est le problème qui ne pèse pas seulement sur une ville ou un pays mais sur le monde entier. Je donne le nom de réchauffement global à ce problème, ce qui est en train d'arriver est, l'excès de $\mathrm{CO} 2$ (gaz carbonique) que les entreprises lancent dans l'air et par conséquent il manque de l'O2 (oxygène) pour l'air. »

12. Selon Nespor \& Vogel (1986), dans des contextes d'énumération, chaque élément configure par lui-même une phrase intonationnelle.

13. Dans les contextes de coordination, pour Nespor \& Vogel (1986), chaque élément de la proposition coordonnée configure une phrase intonationnelle.

14. Nous traduisons le texte portugais suivant : «delimita unidades prosódicas que compõem significativa e formalmente a escrita, com a diferença de que os usos nãoconvencionais são identificados mais por seu caráter icônico para compor os significados enquanto que os usos convencionais são identificados mais por seu caráter formal de organização da escrita. » (Soncin, 2014 : 150)

15. Nous utilisons le terme « iconique " pour caractériser la représentation des attitudes et des émotions du sujet du discours qui composent le rythme du langage, aussi marquée par les signes de ponctuation. Cette interprétation a été donnée par Soncin (2014) à partir des considérations faites par Abaurre (1991) sur l'importance du rythme dans l'acquisition de l'écriture par les enfants. Selon Abaurre (1991), en même temps, le rythme a une signification iconique et une fonction formelle. D'un côté, une signification iconique, parce qu'il marque les émotions et les attitudes du sujet du langage. De l'autre côté, une fonction formelle parce qu'il soutient le matériel phonologique segmental du langage, ce qui lui octroie la condition d'outil pour l'organisation du discours. Abaurre fait la remarque que cette relation iconique entre le rythme et des attitudes et des émotions du sujet souvent nous échappe, parce que l'organisation de l'intonation aussi bien que l'organisation des sons et des gestes est, en général, conventionnée.

\section{RÉSUMÉS}

Cet article vise à montrer comment l'emploi de la virgule peut être interprété comme une marque du processus hétérogène qui caractérise tout acte du langage. En comparant, d'un côté, les normes présentées par des grammairiens du portugais brésilien et, de l'autre côté, les caractéristiques linguistiques et discursives des emplois de la virgule dans les textes d'élèves brésiliens, l'article propose une analyse qui privilégie une discussion concernant les moyens par lesquels les imaginaires sur l'écriture, construits dans le processus de textualisation du discours, constituent l'action symbolique d'employer une virgule dans un texte.

This paper aims to show how the uses of comma can be understood as marks of the heterogeneous process that characterizes every language act. Comparing, on the one hand, the 
rules presented by Brazilian Portuguese grammars and, on the other hand, the linguistic and discursive features observed in the commas used in texts written by Brazilian students, the paper presents an analysis that promotes a discussion about how the imaginary of writing, developed during the process of discourse textualisation, constitutes the symbolic act of using commas in a text.

\section{AUTEURS}

\section{GEOVANA SONCIN}

Université de l'État de São Paulo - UNESP, São José do Rio Preto, São Paulo, Brasil

\section{LUCIANI TENANI}

Université de l'État de São Paulo - UNESP, São José do Rio Preto, São Paulo, Brasil 